\title{
Change in genetic architecture resulting from the evolution of insecticide resistance: a theoretical and empirical analysis
}

\author{
YVES CARRIÈRE* \& DEREK A. ROFF \\ Department of Biology, McGill University, 1205 Dr. Penfield Ave., Montréal, Québec, Canada, H3A $1 B 1$
}

\begin{abstract}
Under selection for insecticide resistance, the spread of a major resistance allele that has strong pleiotropic effects on life history characters will affect the genetic architecture of fitness. A model is developed showing that the spread of such an allele induces a change in the mean and in the additive genetic variance (heritability) of the life history characters, and in the genetic covariance (correlation) between these characters. The model was tested using a quantitative genetic study that compared, in a lepidopteran species (Choristoneura rosaceana), the genetic architecture of diapause propensity and larval weight within and among insecticidefree and insecticide-treated populations from the same geographical area. Significant genetic correlations between resistance to the insecticides and the life history traits were found within the populations, suggesting that the resistance allele(s) has pleiotropic effects on the life history characters. As resistance develops from an initial value of zero, the model predicts a positive relationship between the degree of resistance within the populations and, (1) the magnitude of the fitness costs, (2) the heritability of the life history traits, and (3) the absolute value of the genetic correlations between pairs of life history traits. All these predictions were confirmed. Moreover, the evolution of resistance apparently affected the environmental variance in larval weight. Hence, the novel evolution of insecticide resistance appears to result in major changes in the genetic architecture of fitness, which may limit to some extent the colonization of insecticide-treated habitats.
\end{abstract}

Keywords: diapause, genetic correlation, heritability, insecticide resistance, life history costs, pleiotropy.

\section{Introduction}

In the field, because of the massive doses used, selection for resistance to pesticides often operates at the very extreme of the phenotypic distribution of resistance (Macnair, 1991; McKenzie \& Batterham, 1994). Under such strong selection intensity, genes with major effects are required for individuals to colonize, or to persist in, the pesticide-treated environments (Hedrick \& McDonald, 1980; Wright, 1982; Lande, 1983; Charlesworth, 1990; Macnair, 1991; McKenzie \& Batterham, 1994; Jasieniuk et al., 1995). Accordingly, in the field, the evolution of resistance to insecticides appears to be mostly the result of the action of one or a few genes with major effects, and secondarily of genes with minor effects (Wood, 1981; Roush \& McKenzie, 1987; Tabashnik

\footnotetext{
${ }^{*}$ Correspondence.
}

\& Cushing, 1989; Roush \& Daly, 1990; Mckenzie \& Batterham, 1994; ffrench-Constant, 1994).

Major mutations conferring resistance to insecticides may entail pleiotropic costs that lower the fitness of resistant phenotypes in the absence of insecticide treatments (Abedi \& Brown, 1960; McKenzie et al., 1982; Carrière et al., 1994; Groeters et al., 1994; McKenzie, 1994). Such costs may arise from disruptive effects of the resistance mechanisms on pre-existing functions, or from overproduction of detoxification enzymes diverting energy normally allocated to the life history traits (Uyenoyama, 1986; Lenski, 1988; Roush \& Daly, 1990; Carrière et al., 1994). The change in frequency of a major resistance allele may profoundly influence the genetic properties of a population. First, it will affect the magnitude of the genetic variance in resistance (Falconer, 1989, Chapter 8). Secondly, as will be shown in this paper, if the resistance allele has pleiotropic 
effects on the life history traits, a change in its frequency will necessarily influence the additive genetic variance (heritability) of the life history traits, and hence the genetic covariance (correlation) between them.

The hypothesis of a relationship between the frequency of a resistance allele and the genetic architecture of life history traits has never been tested. Such tests, however, may provide valuable information on the genetic mechanisms involved in the novel adaptation of populations to pesticides, and may serve in the elaboration of measures to prevent or retard the evolution of resistance (Uyenoyama, 1986). Herein, we develop a model that predicts the quantitative genetic consequences on life history traits of variation in the frequency of a major resistance allele. We then test the predictions of this model, by comparing the genetic architecture of diapausing propensity and larval weight among neighbouring populations of the oblique-banded leafroller, Choristoneura rosaceana (Harris), which differed in resistance to synthetic lipophilic insecticides.

\section{The quantitative genetic consequences of the spread of resistance on life history characters}

We assume that insecticide resistance results from the action of a single mutant allele with pleiotropic effects on life history traits. The wild-type allele is designated $M_{1}$ and the mutant allele as $M_{2}$. We shall consider two life history traits, $x$ and $y$, upon which the mutant allele has negative effects, and analyse two cases, the additive model and the dominance model. In particular, we consider an opposite effect of the mutant allele on diapause and larval weight, because the resistant leafrollers were previously found to have smaller larval weight and higher diapausing propensity than the susceptible individuals (Carrière et al., 1994, 1995). We shall assume for modelling purposes that initially the two traits are uncorrelated. This assumption does not alter the qualitative pattern but greatly simplifies the equations. The genotype frequencies and the values of $x$ and $y$ after the mutation of a resistance allele are shown in Table 1. We derive the additive and dominance variances, and heritability for trait $x$, noting that the results for trait $y$ are obtained simply by substituting $-r a$ for $a$ in the formulae.

Let the heritability of $x$ before the mutation be $h_{x}^{2}=V_{\mathrm{A}}(x) / V_{\mathrm{P}}(x)$, where $\mathrm{A}$ and $\mathrm{P}$ refer to the additive and phenotypic components, respectively. For algebraic convenience let $V_{\mathrm{A}}(x)=c_{x} a^{2}$, where $c_{x}$ is a constant. The additive genetic variances resulting
Table 1 Genotypic frequencies and phenotypic values after the mutation of a resistance allele

Phenotypic value of

\begin{tabular}{lccc}
\cline { 3 - 4 } Genotype & Frequency & Trait $x$ & Trait $y$ \\
\hline Additive model & & & \\
$M_{1} M_{1}$ & $p^{2}$ & 0 & 0 \\
$M_{1} M_{2}$ & $2 p q$ & $-a$ & $+r a$ \\
$M_{2} M_{2}$ & $q^{2}$ & $-2 a$ & $+2 r a$ \\
Dominance model & & & \\
$M_{1} M_{1}$ & $p^{2}$ & 0 & 0 \\
$M_{1} M_{2}$ & $2 p q$ & $-a$ & $+r a$ \\
$M_{2} M_{2}$ & $q^{2}$ & $-a$ & $+r a$ \\
\hline
\end{tabular}

The value of $r$ determines the directionality and relative magnitude of the effects of the $M$ locus on the life history traits.

from locus $M, V_{\mathrm{A}}$, are $2 p q a^{2}$ and $8 p^{3} q a^{2}$ for the additive and dominance models, respectively (Falconer, 1989, p. 129). The variances of dominance deviations are likewise 0 and $(2 p q a)^{2}$. After the mutation, the total additive genetic variance is $V_{\mathrm{A}}(x)$ $+V_{\mathrm{A}}=c_{x} a^{2}+V_{\mathrm{A}}$. The total phenotypic variance of $x$ is equal to the original phenotypic variance, $V_{\mathrm{p}}(x)=c_{x} a^{2} / h_{x}^{2}$, plus the additive and dominance contributions from the $M$ locus. Therefore, after the appearance of $M_{2}$ the heritabilities (designated by the subscript $M$ ) are,

additive Model:

$h^{2}{ }_{\mathrm{M} i}=\frac{2 p q+c_{i}}{2 p q+c_{i} / h_{i}^{2}}$

dominance Model:

$h_{\mathrm{M} i}^{2}=\frac{8 p^{3} q+c_{i}}{8 p^{3} q+4 p^{2} q^{2}+c_{i} / h_{i}^{2}}$,

where $i=x$ or $y$. Note that the terms " $a$ " and " $r a$ " in traits $x$ and $y$, respectively, cancel out in the above (their effects are actually absorbed in the terms $c_{x}$ and $c_{y}$ ).

To obtain the genetic correlation between $x$ and $y$ after the mutation, we need to calculate the covariance in breeding values. As $y$ differs from $x$ because of the $M$ locus by the multiplier $-r$, the covariance is equal to $-r$ times the additive genetic variance attributable to $M$ (effects arising from the other loci are independent and therefore do not contribute to the covariance). The genetic correlation is equal to 
Fig. 1 Changes in trait $x$ (in units of $a$ ), in the heritability of traits $x$ and $y$, and in the genetic correlation between them for the additive (left panels) and dominance (right panels) models. For a resistance allele with recessive effects, the changes in heritability and genetic correlation are mirror images of the dominance case. Equations for the heritability and genetic correlation are presented in the text. The change in trait $x$ is given by, (1) additive model: $\Delta x=-2 a q,(2)$ dominance model: $\Delta x=-a q(2-q)$.
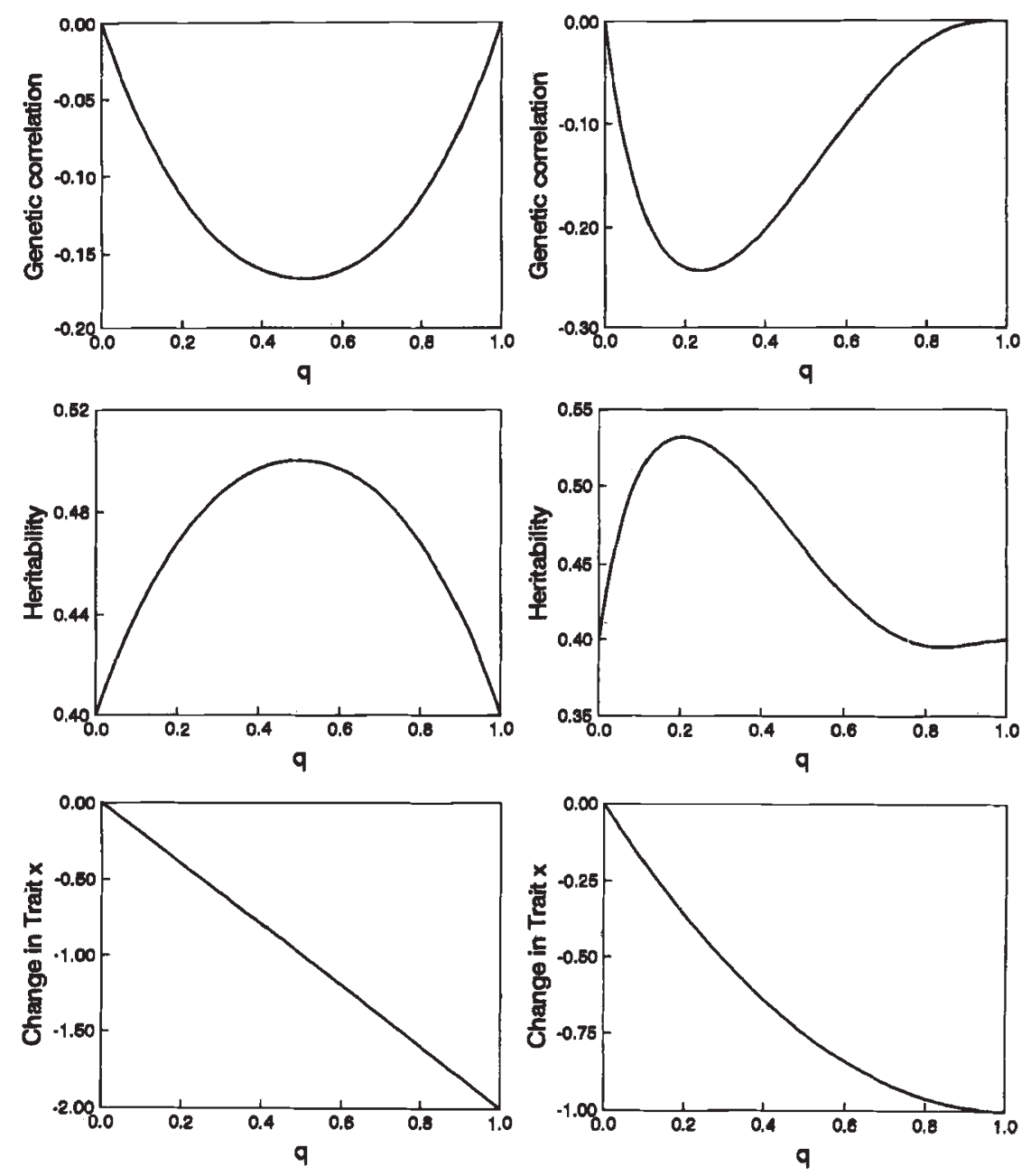

the covariance divided by the square root of the product of the additive genetic variances. Noting that the additive genetic variance of trait $y$ because of the mutation is equal to $r^{2} V_{\mathrm{A}}$, the genetic correlation after mutation for both models can be written as

$r_{\mathrm{A}}=\frac{-r V_{\mathrm{A}}}{\sqrt{\left(V_{\mathrm{A}}+V_{\mathrm{A}}(x)\right)\left(V_{\mathrm{A}} r^{2}+V_{\mathrm{A}}(y)\right)}}$,

where $V_{\mathrm{A}}(y)$ is the additive genetic variance of trait $y$ before the mutation. From the above equation it can be seen that the effect of the mutation is to generate a negative genetic correlation between the two traits. If the two traits were genetically correlated prior to the mutation, the effect of the mutation would be to decrease the genetic correlation. Such a decrease occurs because we assumed an opposite effect of the mutation on the life history traits (i.e. a negative value for $r$ ). However, the spread of a mutation that either increased or decreased both traits would be expected to increase the value of the genetic correlation.

To illustrate the effect of the mutation on the heritabilities and genetic correlations, we present in Fig. 1 a simple example in which we have assumed that before the mutation the heritabilities of both traits are 0.4 , and $c_{x}=c_{y}=r=1$. Note that if the mutant allele is recessive the curve is simply a mirror image of that obtained for the dominance model.

Assuming that the within-population resistance reflects the frequency of the resistance allele(s), the above model suggests that three parameters should change in the leafroller populations as resistance develops from an initial value of zero (Fig. 1): (1) the magnitude of fitness costs (average phenotype) associated with resistance should increase, (2) the 
heritabilities of the life history traits should increase (through a change in the additive components of variance), and (3) the genetic correlations between the life history traits should decrease. In this paper, we will concentrate on the last two predictions, the first having been demonstrated previously (Carrière et al., 1994).

\section{Materials and methods}

\section{Organism and study sites}

The experimental system consists of a generalist herbivorous lepidopteran, the oblique-banded leafroller Choristoneura rosaceana, that is in the process of evolving resistance to synthetic insecticides in apple orchards in the area of Oka, Canada (Carrière et al., 1994, 1995). The oblique-banded leafroller is native to North America (Chapman \& Lienk, 1971) and is present in the deciduous forests of the area of Oka (Carrière, pers. obs.). Prior to the development of resistance, it was sporadically found in low abundance in apple orchards. The discovery of resistance is recent (first detected in 1991) and corresponds with an important increase of its population density in commercial orchards.

The oblique-banded leafroller generally has a partially bivoltine phenology throughout its range (Carrière, 1992, 1994; Carrière et al., 1995). In the area of Oka, every female of the first flight (initiated in mid-June) lays eggs that give rise to larvae that diapause soon after hatching, or that develop and form a second flight occurring in the autumn.

\section{Insect collection and rearing}

Fourth and fifth instar caterpillars were collected in the spring over a period of 10 days from nine insecticide-treated and three insecticide-free orchards (abandoned for more than 10 years) within an area of c. $20 \times 20 \mathrm{~km}$ (described in Carrière et al., 1994, 1995). More than 200 larvae per population yielded adults that provided offspring to establish a first laboratory generation. These adults were obtained and paired as below.

Larvae were raised individually in plastic cups on a standardized pinto bean diet (modified from Shorey \& Hale, 1965) at $22.5 \pm 2^{\circ} \mathrm{C}$ (day) and $20.5 \pm 2^{\circ} \mathrm{C}$ (night) under a 16L:8D photoperiod. Resulting moths were kept in pairs (one male and female). In the first laboratory generation, between 12 and 20 females were raised per population. A family consisted of 50 larvae hatching from the first egg mass of a female. Each family was kept on a tray, and every day the trays were arbitrarily relocated in the growth chamber to minimize microclimatic effects on larval growth.

At least one moth from each family of the first generation was arbitrarily paired to provide offspring to generate a second laboratory generation. Full-sib matings were not allowed, and the number of families raised per population in the second generation was similar to the number raised in the first (range 9-23).

Two life history traits and larval survival to three different insecticides (Azinphosmethyl, AZ; Cypermethrin, CY; and Methomyl, ME) were measured in insects from the second laboratory generation. The proportion of larvae that initiated diapause within each family was estimated in 16-day-old larvae. Diapausing propensity is easily scored at that time because diapausing larvae are much smaller than developing larvae (Carrière, 1992, 1994). In addition, every 16-day-old developing larva was weighted to provide an estimate of growth rate. To measure survival to the insecticides, six developing larvae were arbitrarily selected from each family and exposed to a fixed concentration of a given insecticide (described in Carrière et al., 1995). There were not always enough developing larvae (i.e. 18) to estimate resistance to the three insecticides within each family, which resulted in unequal numbers of families exposed to each insecticide within the populations.

\section{Statistical analysis}

Log-likelihood analyses (PROC CATMOD, SAS, 1988, ML option) were used to test for the presence of significant among-family variation in diapause within each population. The proportion of larvae initiating diapause was treated as a threshold character (Carrière, 1994), and the heritability and standard errors were calculated using the $\chi^{2}$ method for estimation of heritability from family data (Roff, 1986). The additive genetic variance $\left(V_{\mathrm{a}}\right)$ in diapausing propensity was estimated within each population as twice the variance among probit-transformed family values. Such estimates are potentially biased by common environment effects. The families of each population were arbitrarily relocated every day in the growth chamber, however, which implies similar potential bias of $V_{\mathrm{a}}$ in each population. Therefore, the among-population comparisons of $V_{\text {a }}$ should be valid even if $V_{\text {a }}$ may be inflated.

One-way ANOVAS were used to estimate the among-family variation in larval weight within each population (PROC VARCOMP, SAS, 1988). The among-family and environmental components of 
variance were extraced from these analyses (PROC VARCOMP, SAS, 1988), and used to estimate the additive genetic variances (calculated as twice the among-family variance) and heritabilities of larval weight (Falconer, 1989). The standard errors of the heritabilities were estimated according to Becker (1984).

Within-population genetic correlations between the proportion of diapause in a family and the larval weight of its developing members were estimated using family-mean correlations. This method of estimation is approximate as both the genetic covariance and variance may be biased by within-family (environmental) effects (Via, 1991; Roff \& Preziosi, 1995). Such potential contamination, however, is not likely to influence the comparison of the genetic correlations between the insecticide-treated and insecticide-free populations.

Log likelihood analyses (PROC CATMOD, SAS, 1988, ML option) were conducted to test for the presence of significant among-family variation in larval survival to each insecticide within the populations. Family-mean correlations were used to estimate the genetic correlations between survival to each insecticide and the proportion of diapause or larval weight. The genetic correlations were calculated at two levels (Via, 1991); over the entire region (hereafter the 'regional population') and within orchards (hereafter 'within subpopulations'). The family means of the 12 populations were pooled to form the 'regional population' data set. The genetic correlations calculated from this data set indicate genetic constraints for genotypes from the area of Oka in producing phenotypes that would be adapted to both insecticide-treated and insecticidefree habitats (Via, 1991). Genetic correlations were also calculated from a 'within subpopulations' data set containing the residuals from one-way ANOVAS that removed for each character the among-subpopulations phenotypic variation. These correlations are not influenced by differential selection that occurred in the past to generate phenotypic variation among subpopulations. Provided that the resultant genetic correlations result from pleiotropy, they indicate potential constraints on further evolution of resistance within the subpopulations of the area of Oka (Via, 1991). Pooling all family means to calculate the two types of correlations was advantageous because it increased the power of the statistical tests, and was justified because the correlation coefficients calculated for each pair of traits in each of the 12 subpopulations were not statistically different (Sokal \& Rohlf, 1981).

\section{Results}

\section{Heritability of diapause and larval weight}

The propensity to diapause and larval weight had significant heritabilities within each of the 12 populations (Table 2). The average heritability of diapause was $0.42 \pm 0.13( \pm \mathrm{SE})$ in the insecticide-free and $0.77 \pm 0.05$ in the insecticide-treated populations. These heritabilities were significantly different $\left(t_{10}=2.94, P=0.0075\right.$; one-tailed test $)$. The same trend in average heritability of larval weight was observed $(0.56 \pm 0.11$ and $0.65 \pm 0.05$ in the insecticide-free and insecticide-treated populations, respectively), but these values were not significantly different $\left(t_{10}=0.94, P=0.185\right.$; one-tailed test $)$.

Table 2 Heritability estimates within the three insecticidefree and nine insecticide-treated populations of Choristoneura rosaceana

\begin{tabular}{lcc}
\hline Populations & DIAP* & LW* \\
\hline Insecticide-free populations & & \\
H & 0.17 & 0.77 \\
& $(0.072)$ & $(0.22)$ \\
I & 0.47 & 0.38 \\
& $(0.13)$ & $(0.12)$ \\
M & 0.63 & 0.52 \\
& $(0.23)$ & $(0.19)$ \\
Insecticide-treated populations & \\
A & 0.72 & 0.80 \\
& $(0.18)$ & $(0.17)$ \\
B & 0.82 & 0.39 \\
& $(0.31)$ & $(0.19)$ \\
C & 0.52 & 0.57 \\
& $(0.14)$ & $(0.13)$ \\
D & 0.69 & 0.80 \\
& $(0.18)$ & $(0.17)$ \\
E & 0.64 & 0.49 \\
& $(0.16)$ & $(0.14)$ \\
F & 0.71 & 0.66 \\
& $(0.17)$ & $(0.16)$ \\
G & 0.94 & 0.77 \\
& $(0.22)$ & $(0.18)$ \\
J & 0.83 & 0.75 \\
& $(0.20)$ & $(0.17)$ \\
K & 1.04 & 0.67 \\
& $(0.24)$ & $(0.18)$ \\
\hline & &
\end{tabular}

Characters are propensity to diapause (DIAP) and 16-day larval weight (LW). Standard errors are given in parentheses.

*All the within-population family effects were significant $(P<0.05)$. 


\section{Within-population genetic variance in survival to the insecticides}

No larvae survived exposure to $\mathrm{AZ}$ and $\mathrm{CY}$ in two of the three insecticide-free populations, and the variance in survival attributable to the family effect was never significant in populations from nontreated orchards (Table 3). These results indicate low frequencies of the resistance allele(s) in populations not exposed to the insecticides. The among-family variation in survival to $\mathrm{AZ}$ was never significant in the insecticide-treated populations (Table 3). This may result from the high dose of $A Z$ used in the tests that resulted in high mortalities even in the insecticide-treated populations. Such a high dose may have killed even some of the most resistant individuals, thereby reducing the chance of detecting among-family variation in survival to $\mathrm{AZ}$. In contrast, survival to $\mathrm{CY}$ and $\mathrm{ME}$ was high in the insecticide-treated populations, and the among-family variation in resistance was significant in more than 75 per cent of the cases (14/18, Table 3$)$. Therefore, significant genetic variation in resistance to two of the three insecticides appears to be present within the insecticide-treated populations.

Table 3 Significance of the among-family variation in survival to each insecticide within the three insecticidefree and nine insecticide-treated populations of Choristoneura rosaceana

\begin{tabular}{lccc}
\hline Populations & $\mathrm{AZ}$ & $\mathrm{CY}$ & $\mathrm{ME}$ \\
\hline \multicolumn{2}{l}{ Insecticide-free populations } & & \\
$\mathrm{H}$ & $0 \dagger$ & $0 \dagger$ & $2.8^{\mathrm{NS}}$ \\
$\mathrm{I}$ & $1.7^{\mathrm{NS}}$ & $0 \dagger$ & $5.0^{\mathrm{NS}}$ \\
$\mathrm{M}$ & $0 \dagger$ & $3.0^{\mathrm{NS}}$ & $9.7^{\mathrm{NS}}$ \\
\multicolumn{1}{l}{ Insecticide-treated populations } & & \\
$\mathrm{A}$ & $1.7^{\mathrm{NS}}$ & $46.4^{*}$ & $13.1^{*}$ \\
$\mathrm{~B}$ & $1.8^{\mathrm{NS}}$ & $0 \dagger$ & $19.4^{*}$ \\
$\mathrm{C}$ & $1.6^{\mathrm{NS}}$ & $11.1^{*}$ & $20.0^{*}$ \\
$\mathrm{D}$ & $5.9^{\mathrm{NS}}$ & $58.3^{*}$ & $40.7^{\mathrm{NS}}$ \\
$\mathrm{E}$ & $5.2^{\mathrm{NS}}$ & $35.3^{*}$ & $32.3^{*}$ \\
$\mathrm{~F}$ & $8.8^{\mathrm{NS}}$ & $44.9^{*}$ & $48.5^{*}$ \\
$\mathrm{G}$ & $2.4^{\mathrm{NS}}$ & $62.5^{\mathrm{NS}}$ & $76.2^{*}$ \\
$\mathrm{~J}$ & $3.3^{\mathrm{NS}}$ & $10.6^{\mathrm{NS}}$ & $45.0^{*}$ \\
$\mathrm{~K}$ & $10.0^{\mathrm{NS}}$ & $6.7^{*}$ & $22.7^{*}$ \\
\hline
\end{tabular}

Characters are the survival (per cent) to Azinphosmethyl (AZ), Cypermethrin (CY), and Methomyl (ME).

†Among-family variation in survival was not calculated because none of the larvae exposed to the insecticides survived.

${ }^{*} P \leqslant 0.05$.

\section{Relationship between insecticide resistance and the additive genetic variance or heritability of diapause or larval weight}

In previous analyses, it was shown that survival to each insecticide differed significantly between the insecticide-free and insecticide-treated orchards, as well as among the insecticide-treated orchards but not among the insecticide-free orchards (Carrière $e t$ al., 1995). Assuming that the among-orchard variation in resistance reflects differences in the frequency of the resistance allele(s), the correlation between the within-population survival to the insecticides and the additive genetic component of variance or heritability of the life history traits provides a test of the model presented above (see Introduction, prediction 2).

As predicted, the additive component of variance in both diapause and larval weight was positively and significantly correlated with the level of resistance across the 12 populations (Table 4 ). The average $V_{\text {a }}$ for diapause was $0.46 \pm 0.26( \pm S E)$ and $1.18 \pm 0.31$ in the insecticide-free and insecticide-treated populations, respectively. These additive components of variance were significantly different $\left(t_{10}=3.64\right.$, $P=0.0022$; one-tailed test). Likewise, $V_{\mathrm{a}}$ in larval weight was significantly lower in insecticide-free than in insecticide-treated populations $\left(t_{10}=2.33\right.$, $P=0.021 ;$ one-tailed test), with averages of

Table 4 Cross-population correlations between withinpopulation additive genetic component of variance or heritability of the life history traits and insecticide survival

\begin{tabular}{lcccc}
\hline Character & AZ & CY & ME & $X$ \\
\hline Additive genetic variance & & & \\
DIAP & 0.66 & 0.42 & 0.56 & 0.55 \\
& $(0.0095)$ & $(0.090)$ & $(0.029)$ & $(0.030)$ \\
LW & 0.38 & 0.63 & 0.41 & 0.58 \\
& $(0.11)$ & $(0.013)$ & $(0.090)$ & $(0.023)$ \\
Heritability & & & & \\
DIAP & 0.54 & 0.33 & 0.59 & 0.51 \\
& $(0.034)$ & $(0.14)$ & $(0.022)$ & $(0.044)$ \\
LW & 0.17 & 0.56 & 0.41 & 0.52 \\
& $(0.30)$ & $(0.030)$ & $(0.095)$ & $(0.040)$ \\
\hline
\end{tabular}

$\mathrm{AZ}=$ Azinphosmethyl $\mathrm{CY}=$ Cypermethrin;

$\mathrm{ME}=$ Methomyl; $X=$ average survival to the three insecticides.

The life history traits are the propensity to diapause (DIAP) and 16-day larval weight (LW) of Choristoneura rosaceana.

One-tailed levels of significance of the correlation coefficients are given in parentheses. 
$0.36 \pm 0.10( \pm S E)$ and $0.64 \pm 0.07$, respectively. The heritability of diapause and larval weight was posititively and significantly correlated with the level of resistance across the populations (Table 4).

\section{Relationship between insecticide resistance and the environmental component of variance in larval weight}

Interestingly, the environmental component of variance in larval weight was significantly higher in the insecticide-treated $(0.65 \pm 0.026[ \pm \mathrm{SE}])$ than in the insecticide-free populations $\left(0.47 \pm 0.052 ; t_{10}=3.25\right.$, $P=0.0087$; two-tailed test). This increase ( 38 per cent), however, was much smaller than the increment in the additive genetic component of larval weight (78 per cent). Moreover, the environmental component of variance in larval weight was not significantly correlated with the degree of resistance to the insecticides (two-tailed tests; AZ: $r=0.52$, $P=0.080 ; \mathrm{CY}: r=0.24, P=0.45 ; \mathrm{ME}: r=0.14$, $P=0.65 ; X: r=0.24, P=0.45)$. Therefore, the predictions of the model on the variation in the genetic architecture of the life history traits should still be valid, despite the assumption of a constant environmental variance between the insecticide-free and insecticide-treated populations.

Table 5 Within-population genetic correlations between the propensity to diapause within a family of Choristoneura rosaceana and the larval weight of its members

\begin{tabular}{lccr}
\hline Population & $r$ & $P$ & $N$ \\
\hline Insecticide-free populations & & \\
H & -0.58 & 0.0460 & 12 \\
I & -0.49 & 0.0280 & 20 \\
M & -0.41 & 0.1800 & 12 \\
Insecticide-treated populations & & \\
A & -0.85 & 0.0001 & 21 \\
B & -0.60 & 0.0860 & 9 \\
C & -0.62 & 0.0028 & 21 \\
D & -0.58 & 0.0087 & 19 \\
E & -0.81 & 0.0001 & 21 \\
F & -0.63 & 0.0011 & 23 \\
G & -0.73 & 0.0004 & 19 \\
J & -0.71 & 0.0003 & 21 \\
K & -0.85 & 0.0001 & 19 \\
\hline
\end{tabular}

$P$ is the level of significance of the correlation coefficients and $N$ is the number of families involved in the calculations.

\section{Genetic correlations between diapause and larval weight}

As predicted, the genetic correlations between diapause and larval weight were more negative in the insecticide-treated $(-0.71 \pm 0.04[ \pm \mathrm{SE}])$ than in the insecticide-free populations $(-0.49 \pm 0.05)$ (Table 5; $t_{10}=3.12, P=0.0055$; one-tailed test).

\section{Genetic correlations between insecticide resistance and diapause or larval weight}

The genetic correlations calculated within the 'regional population' revealed significant positive correlations between diapause and resistance to $\mathrm{CY}$ or ME, but the correlation was not significantly different from zero between diapause and survival to $\mathrm{AZ}$ (Table 6). Likewise, the genotypes that were most resistant to $\mathrm{CY}$ and $\mathrm{ME}$ had lower growth rates (Table 6), although this association was not found between resistance to $\mathrm{AZ}$ and larval weight. The partial diapause in the oblique-banded leafroller can be viewed as a bet-hedging strategy that evolved to minimize the risk of reproductive failure in unpredictable environments (Carrière et al., 1995). Provided that the insecticide-free populations

Table 6 Genetic correlations for the 'regional population' and 'within subpopulations' between the propensity to diapause (DIAP) or 16-day larval weight (LW) of Choristoneura rosaceana and the proportion of survival to each insecticide

\begin{tabular}{|c|c|c|}
\hline Insecticide survival & DIAP & LW \\
\hline \multicolumn{3}{|l|}{ 'Regional population' } \\
\hline \multirow[t]{2}{*}{$\mathrm{AZ}$} & $0.080(0.27)$ & $0.0074(0.92)$ \\
\hline & 193 & 193 \\
\hline \multirow[t]{2}{*}{ CY } & $0.24(0.007)$ & $-0.23(0.010)$ \\
\hline & 129 & 129 \\
\hline \multirow[t]{2}{*}{ ME } & $0.37(0.0001)$ & $-0.48(0.0001)$ \\
\hline & 138 & 138 \\
\hline \multicolumn{3}{|c|}{ 'Within subpopulations' } \\
\hline \multirow[t]{2}{*}{$\mathrm{AZ}$} & $-0.021(0.77)$ & $0.14(0.040)$ \\
\hline & 193 & 193 \\
\hline \multirow[t]{2}{*}{ CY } & $0.022(0.80)$ & $0.0041(0.96)$ \\
\hline & 129 & 129 \\
\hline \multirow[t]{2}{*}{$\mathrm{ME}$} & $0.23(0.0067)$ & $-0.31(0.0002)$ \\
\hline & 138 & 138 \\
\hline
\end{tabular}

$\mathrm{AZ}=$ Azinphosmethyl $\mathrm{CY}=$ Cypermethrin; $\mathrm{ME}=$ Methomyl.

Levels of significance are given in parentheses and the numbers of families involved in the calculations appear under the coefficients. 
are near the optimal diapausing propensity corresponding to the level of unpredictability in the area of Oka, the higher diapause of the resistant genotypes in the untreated habitats will reduce their rate of increase compared to the susceptible genotypes. Therefore, given that the 12 populations are representative of the area, these results suggest that the evolution of resistance to $\mathrm{CY}$ and $\mathrm{ME}$ involved fitness costs, taking the form of higher diapause and lower growth rate for the resistant genotypes that colonize untreated habitats.

The 'within-subpopulations' genetic correlations indicate potential pleiotropic effects of resistance allele(s) that may constrain further evolution of resistance. Three significant correlations were found: between resistance to $\mathrm{AZ}$ and larval weight, between resistance to $\mathrm{ME}$ and larval weight, and between resistance to $\mathrm{ME}$ and diapause propensity (Table 6). The positive genetic correlation between resistance to $\mathrm{ME}$ and diapause suggests that further increase in resistance to ME may result in higher diapause in insecticide-treated orchards. Such an increase in resistance to ME would also result in a reduction of larval growth rate (Table 6). The positive genetic correlation between survival to $\mathrm{AZ}$ and larval weight has to be taken cautiously, because survival to $\mathrm{AZ}$ was very low and had a nonsignificant heritability (Table 3). It suggests, however, that a simultaneous increase in resistance to $\mathrm{AZ}$ and $\mathrm{ME}$ may have opposite indirect effects on the evolution of growth rates.

\section{Discussion}

The spread of a major mutation with negative pleiotropic effects will progressively lower the average fitness of individuals raised in the absence of insecticides. Once such a mutation becomes common within a population, however, the fitness costs associated with resistance may be alleviated if the original resistance allele is replaced by a less harmful mutation, or if a modifier allele that reduces the deleterious effects of the original mutation increases in frequency (Abedi \& Brown, 1960; Wright, 1977; Wood \& Bishop, 1981; McKenzie et al., 1982; Uyenoyama, 1986; Lenski, 1988; McKenzie \& O'Farrell, 1993; Cohan et al., 1994). In this paper, we are concerned with the first phase of the development of resistance that involves the initial increase in frequency of a major resistance allele, rather than with the second phase where a genomic rearrangement may be taking place. We hypothesized that the spread of major mutations with pleiotropic effects that initially confer resistance to insecticides would influence the genetic architecture of fitness.

Our model predicted that the frequency of the resistance allele would affect the mean and the heritability (additive genetic variance) of the life history characters, and the genetic correlation between them. The first prediction of the model was supported, there being strong positive across-population correlations between resistance and diapausing propensity (Carrière et al., 1995) and between resistance and development time, and strong negative correlations between resistance and 16-day larval weight or pupal weight (Carrière et al., 1994). Moreover, the additive genetic component of variance in diapause or larval weight and the heritability of diapause increased, whereas the genetic correlation between diapause and larval weight decreased substantially in the insecticide-treated populations (Tables 4 and 5). Finally, significant positive correlations were found between the additive genetic component of variance or heritability of the life history traits and the degree of resistance. Such quantitative genetic changes suggest that the spread of the resistance allele(s) caused local increases in pleiotropic effects on diapause and larval weight. The presence of pleiotropic effects of the resistance allele(s) on the fitness components is indicated by the significant 'within-subpopulations' genetic correlations (Table 6). Therefore, the results of this study support the hypothesis that the evolution of insecticide resistance involving pleiotropic costs has substantial consequences on the genetic architecture of fitness. In what follows, we discuss the implications of these findings, and the possible role of alternative mechanisms in generating the change in the genetic architecture of the life history traits.

Assuming no successful migration of the susceptible genotypes into the insecticide-treated orchards, the increase in additive genetic variance (heritability) of the life history traits provides an estimate of the pleiotropic costs associated with resistance. For life history traits, the additive genetic variance alone will determine the response to selection (Falconer, 1989 , p. 346). Therefore, the magnitude of the increase in the additive genetic variance of the life history traits in the resistant populations would reflect their rate of reversal toward susceptibility in the absence of insecticide treatments (Uyenoyama, 1986). Hence, it is possible to use our model to predict the rate of reversal to susceptibility in the absence of insecticide for cases where the resistance allele has recessive, additive, or dominant effects on fitness. The rate of reversal is expected to be minimal at a low frequency of the resistance allele (when 
the heritability of the life history traits is moderately inflated), and to be maximal when a frequency of $0.8,0.5$, and 0.2 is reached for the recessive, additive, and dominant resistance allele, respectively (Fig. 1). Therefore, when the resistance allele is at low frequency within the populations of a region, resistance should generally reverse at a faster rate in the most resistant than in the less resistant populations. When the frequency of the resistance allele approaches 1 within such populations, however, the heritability of the life history traits will decline to the values that were present before the emergence of resistance (Fig. 1). Then, resistance may generally reverse at a faster rate in populations with intermediate rather than high resistance (i.e. reversal may be faster in populations where the heritability of the life history traits is maximally inflated).

Inspection of the plots relating the additive genetic variance of larval weight or diapause to average resistance suggests that the frequency of the resistance allele(s) at which the effect on the genetic architecture of the life history traits is maximal was reached within the populations of this system (Fig. 2 ). Indeed, the fit of the relationship between $V_{\mathrm{a}}$ and resistance was improved significantly by the addition of a quadratic term in the case of larval weight ( $t_{9}=-2.077, P=0.034$; one-tailed test), and marginally in the case of diapause $\left(t_{9}=-1.693\right.$, $P=0.062$ ). Nevertheless, the decline in $V_{\mathrm{a}}$ in the most resistant populations was not very pronounced (Fig. 2), suggesting that resistance would still reverse at a faster rate in the most resistant populations of this system, provided that pesticides which do not discriminate between resistant and susceptible phenotypes were used to control this insect.

Faster rates of reversal to susceptibility in the most resistant populations were observed in the mosquito Anopheles culicifacies after withdrawal of DDT spraying (Curtis et al., 1978). Assuming that the migration rate of susceptibles in treated areas was not related to the level of resistance, Curtis et al. (1978) suggested that this phenomenon could be explained by, (1) balancing selection favouring resistant heterozygotes, or (2) the presence of two different resistance mechanisms conferring low and high resistance with proportional fitness costs. This phenomenon could also be explained by the model developed in the present paper.

Major mutations conferring resistance to insecticides may affect developmental stability and result in increased levels of fluctuating asymmetry in the resistant genotypes (McKenzie \& Clarke, 1988; McKenzie \& O'Farrell, 1993). Such developmental instability may result from interference of the novel
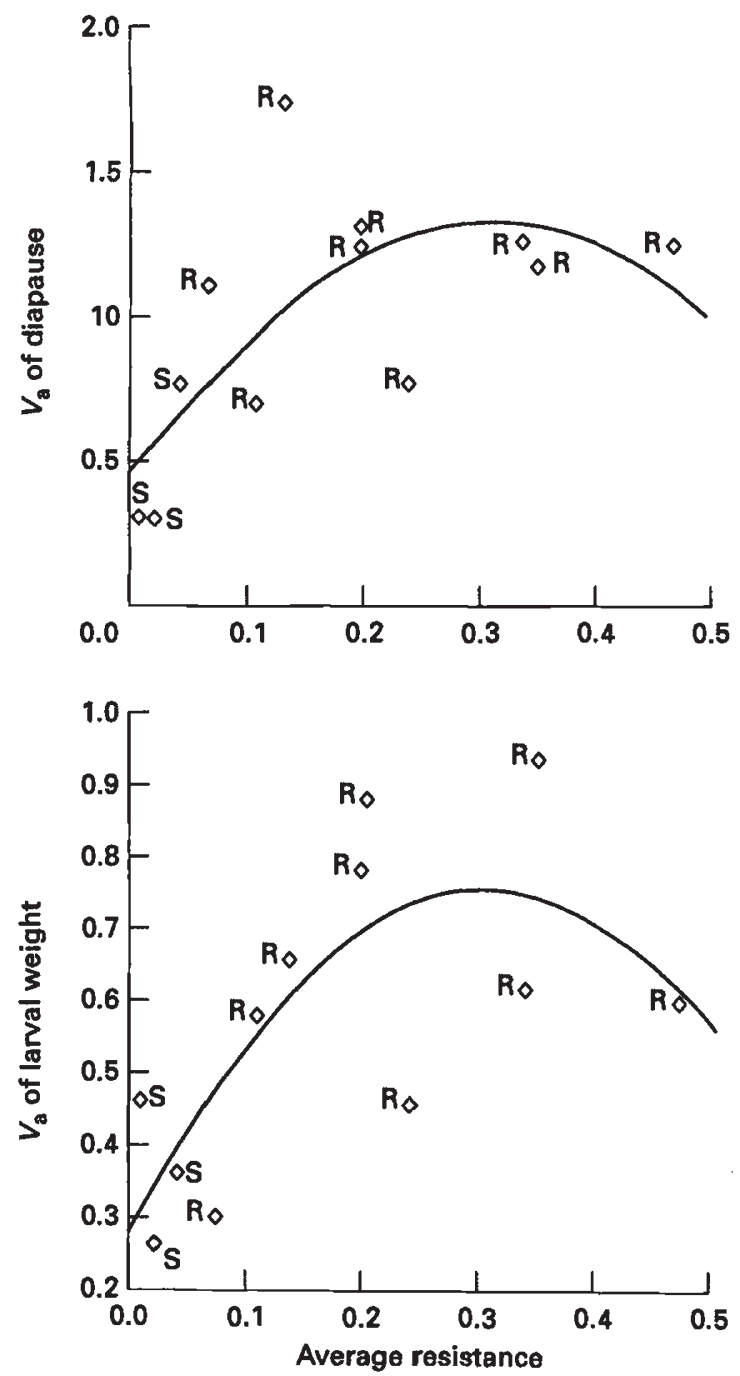

Fig. 2 Relationship between the additive genetic variance in diapause (top) or larval weight (bottom) and the average resistance to insecticides within insecticide-free $(\mathrm{S})$ and insecticide-treated $(\mathrm{R})$ populations.

resistance functions with normal physiological processes that reduce the ability of genotypes to canalize development in the presence of environmental sources of variance. The increase in the environmental component of variance in larval weight in the insecticide-treated populations of $C$. rosaceana may be another manifestation of the disruptive effects of the resistance allele(s). Such an effect, if deleterious, could be eliminated by fixation of modifier alleles following continuous selection for insecticide resistance, as was observed in the case of fluctuating asymmetry in Lucilia cuprina (McKenzie \& O'Farrell, 1993).

This study has important theoretical and practical implications. It illustrates a mechanism that can limit

(c) The Genetical Society of Great Britain, Heredity, 75, 618-629. 
evolutionary responses of populations by natural selection. In British Columbia, Canada where resistance of the oblique-banded leafroller to insecticide has not yet appeared, $C$. rosaceana is extremely susceptible to synthetic insecticides and is almost totally eliminated following a single application of Azinphosmethyl (Carrière, pers. obs.). Selection for insecticide resistance appears to operate at the very extreme of the phenotypic distribution of resistance (McKenzie \& Batterham, 1994). In Oka, however, population densities remain relatively large even if the leafrollers are exposed to insecticides (especially to $\mathrm{AZ}$ and CY). The spread of resistance allele(s) has made accessible a novel adaptive peak. Accordingly, both major and minor mutations can now contribute to the evolution of the resistant phenotypes in this novel habitat (Lande, 1983). The spread of the resistance allele(s), however, apparently resulted in local increases in pleiotropic costs affecting the fitness components, which is one element that may impose limits on evolution (Antonovics, 1976; Bradshaw, 1991), especially in the absence of strong directional selection (Wright, 1982; Lande, 1983; Lenski, 1988). This study also provides a basis for understanding how the genetic properties of populations vary during the transient period over which the resistance allele(s) increases toward fixation. Our results suggest that novel insecticides that do not discriminate between resistant and susceptible genotypes should be implemented before the resistance allele(s) becomes too abundant. This will prevent the decline in the potential of the resistant populations to reverse toward susceptibility, which should maximize the chances of reversing the evolution of resistance to insecticides.

The change in mean and genetic architecture of the life history traits could result from variation in the frequency of the resistance allele(s) that induced local changes in pleiotropic effects. However, we cannot at this point eliminate the possibility that population bottlenecks in the insecticide-treated populations resulted in inbreeding depression (Charlesworth \& Charlesworth, 1987; Falconer, 1989), and in increased additive variance caused by random changes in the frequency of recessive deleterious alleles (Robertson, 1952; Willis \& Allen Orr, 1992), or by conversion of epistatic genetic variance into additive variance (Goodnight, 1988). However, bottleneck effects are unlikely to have been important in this system for three reasons. First, the contribution of nonadditive genetic variance to the phenotypic variation in diapause and development time does not appear to be important in $C$. rosaceana (Carrière, 1994, unpublished data). Secondly, bottleneck effects would need to be proportional to the degree of insecticide resistance to explain the changes in the life history traits observed in this study. Thirdly, the direction of the change in diapause and larval weight was the same in every insecticide-treated subpopulation (Carrière et al., 1994, 1995), and the additive genetic variance of these traits never decreased (see Fig. 2), which is not expected for a group of subpopulations that diverged because of genetic drift (Falconer, 1989; Mitchell-Olds, 1990).

As previously mentioned, common environment effects may have inflated the heritability estimates, although this is unlikely to explain the systematic differences among the subpopulations. Gene flow between subpopulations exploiting deciduous forests and insecticide-treated orchards may also have contributed to inflation of the heritabilities of the life history traits in the insecticide-treated habitats, or to making the genetic correlation between diapause and larval weight more negative in the treated than in the nontreated orchards (susceptible genotypes have higher larval weight and lower diapause than resistant genotypes). Gene flow could also have influenced the 'within-subpopulations' genetic correlations between resistance and the life history traits through linkage disequilibrium. Different rates of approach to linkage equilibrium following two generations of random mating would then have to be invoked to explain why significant 'within-subpopulations' genetic correlations were not detected between resistance to each of the three insecticides and the life history traits. Further research is needed to verify the importance of pleiotropy and to exclude the effects of bottlenecks or gene flow in this system.

\section{Acknowledgements}

We thank J.-P. Deland for outstanding assistance and enthusiasm in conducting these experiments. We are also grateful to the staff of the Saint-Jeansur-Richelieu Agriculture Canada Research Station for their collaboration. This research was supported by a grant from the Natural Sciences and Engineering Research Council of Canada to D. Roff and post-doctoral scholarships from NSERC and Les Fonds de Soutien à la Recherche du Québec to Y. Carrière.

\section{References}

ABEDI, Z. H. AND BRowN, w. A. 1960. Development and reversion of DDT-resistance in Aedes aegypti. Can. J. Genet. Cytol., 2, 252-261. 
ANTONOVICs, J. 1976. The nature of limits to natural selection. Ann. Mo. Bot. Gard., 63, 224-247.

BECKER, w. A. 1984. Manual of Quantitative Genetics. Academic Enterprise, Pullman, WA.

BRADSHAW, A. D. 1991. The Croonian Lecture. Genostasis and the limits to evolution. Phil. Trans. R. Soc. B, 33, 289-305

CARriÈre, Y. 1992. Host plant exploitation within a population of a generalist herbivore, Choristoneura rosaceana. Entomologia exp. appl., 65, 1-10.

CARRIÈRE, Y. 1994. Evolution of phenotypic variance: nonMendelian parental influences on the phenotypic and genotypic components of the life-history traits in a generalist herbivore. Heredity, 72, 420-430.

CARRIÈRE, Y., DELAND, J.-P., ROFF, D. A. AND VINCENT, C. 1994. Life history costs associated with the evolution of insecticide resistance. Proc. $R$. Soc. B, 258, 35-40.

CARRIÈrE, Y., ROFF, D. A. AND DELAND, J.-P. 1995. The joint evolution of diapause and insecticide resistance: a test of an optimality model. Ecology, 76, 1497-1505.

CHAPMAN, P. J. AND LIENK, s. E. 1971. Tortricid Fauna of Apple in New York. Special publication. New York. Agric. Exp. Stn., Geneva, NY.

CHARlESWORTH, B. 1990. The evolutionary genetics of adaptation. In: Niteckie, M. H. (ed.) Evolutionary Innovations, pp. 47-70. University of Chicago Press, Chicago.

CHARLESWORTH, B. AND CHARLESWORTH, D. 1987. Inbreeding depression and its evolutionary consequences. Ann. Rev. Ecol. Syst., 18, 237-268.

COHAN, F. M., KING, E. C. AND ZAWADZKI, P. 1994. Amelioration of the deleterious pleiotropic effects of an adaptive mutation in Bacillus subtilis. Evolution, 48, 81-95.

CURTIS, C. F., COOK, L. M. AND WOOD, R. J. 1978. Selection for and against insecticide resistance and possible methods of inhibiting the evolution of resistance in mosquitoes. Ecol. Entomol., 3, 273-287.

FALCONER, D. S. 1989. Introduction to Quantitative Genetics, 3rd edn. Longman, New York.

FFRENCH-CONSTANT, R. H. 1994. The molecular and population genetics of cyclodiene insecticide resistance. Insect Biochem. Mol. Biol., 4, 335-345.

GOODNIGHT, C. 1988. Epistasis and the effect of founder events on epistatic genetic variance. Evolution, 41, 80-91.

GROETERS, F. R., TABASHNIK, B. E., FINSON, N. AND JOHNSON, M. W. 1994. Fitness costs of resistance to Bacillus thuringiensis in the diamondback moth (Plutella xylostella). Evolution, 48, 197-201.

HEDRICK, P. W. AND McDONALD, J. F. 1980. Regulatory gene adaptation: an evolutionary model. Heredity, 45, 83-97.

JASIENIUK, M., BRULE-BABEL, A. L. AND MORRISON, I. N. 1995. The evolution and genetics of herbicide resistance in agricultural weeds. Weed Sci., (in press).

LANDE, R. 1983. The response to selection on major and minor mutations affecting a metrical trait. Heredity, $\mathbf{5 0}$, $47-65$.

LENSKI, R. E. 1988. Experimental studies of pleiotropy and epistasis in Escherichia coli. II. Compensation for maladaptive effects associated with resistance to virus $\mathrm{T} 4$ Evolution, 42, 433-440.

MACNAIR, M. R. 1991. Why the evolution of resistance to anthropogenic toxins normally involves major gene changes: the limits to natural selection. Genetica, 84, 213-219.

McKENZIE, J. A. 1994. Selection at the diazinon resistance locus in overwintering populations of Lucilia cuprina (the Australian sheep blowfly). Heredity, 73, 57-64.

McKenZiE, J. A. AND BATTERhAM, P. 1994. The genetic, molecular and phenotypic consequences of selection for insecticide resistance. Trends Ecol. Evol., 9, 166-169.

McKENZIE, J. A. AND ClARKE, G. M. 1988. Diazinon resistance, fluctuating asymmetry and fitness in the Australian sheep blowfly, Lucilia cuprina. Genetics, 120, 213-220.

McKenZIE, J. A. AND O'FARRELL, K. 1993. Modification of developmental instability and fitness: malathion-resistance in the Australian sheep blowfly, Lucilia cuprina. Genetica, 89, 67-76.

McKenziE, J. A., WhitTen, M. J. AND ADENA, M. A. 1982. The effect of genetic background on the fitness of diazinon resistance genotypes of the Australian sheep blowfly, Lucilia cuprina. Heredity, 49, 1-9.

MITCHELL-OLDS, T. 1990. Quantitative genetic changes in small populations. In: Dudley, E. C. (ed.) The Unity of Evolutionary Biology, vol II, pp. 634-638. Dioscorides Press, Portland, OR.

ROBERTSON, A. 1952. The effect of inbreeding on the variation due to recessive genes. Genetics, 37, 189-207.

ROFF, D. A. 1986. The genetic basis of wing dimorphism in the sand cricket, Gryllus firmus and its relevance to the evolution of wing dimorphisms in insects. Heredity, 57, 221-231.

ROFF, D. A. AND PREZIOS1, R. 1995. The estimation of the genetic correlation: the use of the jacknife. Heredity, 73, 544-548.

ROUSH, T. R. AND DALY, J. C. 1990. The role of population genetics in resistance research and management. In: Roush, R. T. and Tabashnik, B. E. (eds) Pesticide Resistance in Arthropods, pp. 97-152. Chapman and Hall, New York.

ROUSH, T. R. AND McKENZIE, J. A. 1987. Ecological genetics of insecticide and acaricide resistance. Ann. Rev. Ent., 32, 361-380.

SAS INSTITUTE. 1988. SAS/sTAT User's guide, Release 6.03 Edition. SAS Institute, Cary, NC.

SHOREY, H. H. AND HALE, R. C. 1965. Mass rearing of the larvae of nine noctuid species on a simple artificial medium. J. Econ. Entomol., 58, 522-524.

SOKAL, R. R. AND ROHLF, R. J. 1981. Biometry, 2nd edn. Freeman and Co., New York.

TABASHNIK, B. E. AND CUSHING, N. L. 1989. Quantitative genetics analysis of insecticide resistance: Variation in Fenvalerate tolerance in a diamondback moth (Lepidoptera: Plutellidae) population. J. Econ. Entomol., 82, 5-10.

UYeNoyAmA, M. K. 1986. Pleiotropy and the evolution of 
genetic systems conferring resistance to pesticides. In: Glass, E. H. (chairman) Pesticide Resistance: Strategies and Tactics for Management, pp. 207-221. National Academy of Sciences, Washington, DC.

VIA, s. 1991. The genetic structure of host plant adaptation in a spatial patchwork: demographic variability among reciprocally transplanted pea aphid clones. Evolution, 45, 827-852.

wILLIS, J. H. AND ALLEN ORR, H. 1992. Increased heritable variation following population bottlenecks: the role of dominance. Evolution, 47, 949-957.

wOOD, R. J. 1981. Insecticide resistance: gene and mechan- isms. In: Bishop, J. A. and Cook, L. M. (eds) Genetic Consequences of Man-made Changes, pp. 53-96. Academic Press, New York.

WOOD, R. J. AND BISHOP, J. A. 1981. Insecticide resistance: population and evolution. In: Bishop, J. A. and Cook, L. M. (eds) Genetic Consequences of Man-made Changes, pp. 97-127. Academic Press, New York.

WRIGHT, s. 1977. Evolution and the Genetics of Populations, vol. 3, Experimental Results and Evolutionary Deductions. University of Chicago Press, Chicago.

WRIGHT, s. 1982. Character change, speciation, and the higher taxa. Evolution, 36, 427-443. 